\title{
Salud y Regímenes Políticos: ¿Presidencialismo o Parlamentarismo para Colombia?
}

\author{
Heath and political regimes: Presidential or parliamentary \\ government for Colombia?
}

\author{
Alvaro J. Idrovo \\ Universidad Autónoma de Chihuahua. Chihuahua, México.idrovoaj@yahoo.com.mx \\ Recibido 27 Julio 2006/Enviado para Modificación 12 Diciembre 2006/Aprobado 14 Marzo 2007
}

\section{RESUMEN}

Objetivo En Colombia se discute actualmente la posibilidad de cambiar su régimen presidencial por uno parlamentario. Este estudio preliminar explora los potenciales efectos sobre la salud de los regímenes presidencial y parlamentario con datos mundiales.

Material y métodos Se realizó un estudio ecológico con datos de los países de los que se obtuvo información comparable para esperanza de vida al nacer, régimen político, desarrollo económico, desigualdad en el ingreso, capital social [medido con la confianza generalizada y la corrupción percibida], derechos políticos y libertades civiles, y diversidad cultural. Se comparó la esperanza de vida al nacer y los macrodeterminantes entre ambos regímenes. Las correlaciones entre estos macrodeterminantes fueron estimadas, y luego se exploró la relación entre el régimen político y la esperanza de vida al nacer mediante regresiones robustas.

Resultados En el análisis crudo se observó que los países con régimen parlamentario tienen una mayor esperanza de vida al nacer que los que tienen régimen presidencial. Se observaron correlaciones significativas entre todos los macrodeterminantes. En las regresiones robustas múltiples no se observó un efecto diferencial entre los dos regímenes políticos sobre la esperanza de vida al nacer.

Discusión No hay evidencia de que un régimen presidencial o parlamentario provea mayores niveles de salud a la población. Se sugiere enfocar las políticas de salud pública a otros macro-determinantes con efecto más conocido sobre la salud como la desigualdad en el ingreso.

Palabras Clave: Política, políticas públicas, Colombia (fuente: DeCS, BIREME).

\section{ABSTRACT}

Objective Changing the presidential regime for a parliamentarian one is currently being discussed in Colombia. This preliminary study explores the potential effects on 
health of both presidential and parliamentary regimes by using world-wide data. Material and methods An ecological study was undertaken using countries from which comparable information concerning life-expectancy at birth, political regime, economic development, inequality in income, social capital (as measured by general-ised trust or Corruption Perceptions Index), political rights, civil freedom and cultural diversity could be obtained. Life-expectancy at birth and macro-determinants were compared between both political regimes. The co-relationship between these macro-determinants was estimated and the relationship between political regimen and life-expectancy at birth was estimated using robust regression.

Results Crude analysis revealed that parliamentary countries have greater lifeexpectancy at birth than countries having a presidential regime. Significant corelationships between all macro-determinants were observed. No differential effects were observed between both political regimes regarding life-expectancy at birth in multiple robust regressions.

Discussion There is no evidence that presidential or parliamentary regimes provide greater levels of health for the population. It is suggested that public health policies be focused on other macro-determinants having more known effects on health, such as income inequality.

Key Words: Politics, public policy, Colombia (source: $M e S H, N L M$ ).

$\mathrm{E}$

n los meses previos se ha venido hablando de manera insistente acerca de la posibilidad de que Colombia cambie su régimen presidencial por uno parlamentario. Los que sostienen esta postura se fundamentan en argumentos como que los regímenes presidenciales son más rígidos e inflexibles, tienden a excluir a los grupos que no están en el gobierno $(1,2)$, son menos estables y son más sensibles a la heterogeneidad religiosa y de partidos políticos (3). Las ventajas del régimen parlamentario tendían a ser un consenso hasta cuando Shugart \& Carey (4), y después otros autores, mostraron evidencias de que el régimen presidencial per se no es inestable sino que es dependiente de características especiales de la forma en que se ejerce el poder presidencial. Como se puede apreciar, el tema resulta complejo y existen datos empíricos contradictorios, por lo que es mejor dejar el tema para los especialistas.

Sin embargo, para quienes interesa la salud de los colombianos, tanto a nivel individual como poblacional, resulta importante conocer qué efectos positi-vos y/o negativos se han visto potencialmente asociados con estos regímenes. Este tipo de temas ha sido motivo de exploración reciente por la epidemiología social. Se ha descrito que la democracia tiene un efecto positivo sobre la salud, después de ajustar por el desarrollo económico, el nivel de desigualdad y el tamaño del sector público (5) y que su efecto depende de la ubicación del país en el mercado mundial (6) y el capital social entre otros 
determinantes $(7,8)$. Sin embargo, de manera explícita no se ha hecho un estudio que compare los regímenes presidencial y parlamentario, por lo que el objetivo de este análisis preliminar es explorar los potenciales efectos del régimen político presidencial o parlamentario sobre la esperanza de vida al nacer (EVN), utilizando los datos mundiales comparables disponibles.

\section{MATERIALES Y MÉTODOS}

Diseño y evento en salud

Se realizó un análisis ecológico exploratorio (9) con todos los países de los que se obtuvo información comparable del régimen político, presidencial o parlamentario, y de otros macro-determinantes conocidos. El evento de interés fue la $\mathrm{EVN}$, que puede definirse como el número promedio de años adicionales que una persona podría vivir si las tendencias en la mortalidad actuales continuaran por el resto de su vida (10). Este indicador fue seleccionado ya que resulta ser uno de los pocos que intenta captar el concepto de salud y no de enfermedad (11). Los datos utilizados en el análisis fueron tomados del Informe de la Organización Mundial de la Salud 2004 (12). Al seleccionar la EVN como evento de estudio se evita el problema de no considerar un periodo de latencia entre la exposición y el evento en salud, ya que la EVN refleja en gran medida la mortalidad infantil que se puede asociar mejor con las condiciones contemporáneas (8).

\section{Evaluación del régimen político}

Para este análisis preliminar se utilizaron los datos disponibles en un documento de trabajo de Lawrence (13), modificado a su vez de un estudio previo (14). En este documento se encuentran los regímenes presiden-cial, parlamentario y mixtos en diferentes periodos, pero sólo se inclu-yeron en el análisis los regímenes presidencial y parlamentario. En es-te trabajo se calificó un régimen como "presidencial" si el presidente fue efectivamente el jefe del poder ejecutivo del país (13). Únicamen-te los países que no presentaron cambios de régimen entre 1950 y 1990 fueron incluidos en este análisis.

Otras variables

El desarrollo económico fue medido con el Producto Interno Bruto (PIB) ajustado por la paridad de poder de compra (ppc), reportado en el Informe Mundial de 2004 del Programa de las Naciones Unidas para el Desarrollo 
(15). El PIB/ppc fue seleccionado ya que el PIB crudo no permite hacer comparaciones internacionales, y para los análisis se usó su logaritmo ya que es bien conocida su distribución altamente asimétrica (6). Este indicador debe interpretarse como que a mayor PIB/ppc mayor desarrollo económico. La desigualdad en el ingreso fue medida con el coeficiente de Gini, el cual es su forma más frecuente de medición; éste presenta valores entre cero y uno, siendo mayor cuando más concentración de la riqueza hay y menor cuando es más igualitaria la distribución del ingreso (16). Los datos utilizados aquí fueron obtenidos del Informe Mundial de Salud de 2004 (15). Para fines de los análisis los datos originales fueron convertidos a una escala de cero a 100, conservando la misma interpretación.

El capital social fue medido mediante la confianza generalizada y la corrupción percibida; el primero es un indicador directo y es la forma más usual de medir el capital social, aunque presenta como limitante que sólo un limitado número de países cuenta con información comparable de este tipo. La corrupción percibida es un indicador indirecto, hace poco incorporado en-tre las formas de medir el capital social $(17,18)$. El nivel de confianza gene-ralizada de cada país fue tomado de un estudio previo, en el que se evaluó el capital social usando las Encuestas Mundiales de Valores (World Value Sur-veys). En éstas encuestas se encuentran los aspectos de confianza generaliza-da, normas sociales y participación voluntaria en organizaciones.

En este estudio sólo se usa el porcentaje de la población que respondió afirmativamente a la pregunta: "En general, ¿usted piensa que no se debe confiar en la mayoría de la gente, o si es el caso debe serse muy cuidadoso? (17). En este indicador cuando más altos son los valores se asocia con un mayor nivel de capital social. La corrupción prevalente en cada uno de los países participantes fue determinada mediante el Indice de Percepción de Corrupción 2004 de Transparency International (19). Este es un índice que oscila entre cero y 10, siendo menor entre más corrupción se perciba, que in-corpora información entre 2002 y 2004 de por lo menos tres fuentes diferen-tes de 18 sondeos y encuestas realizados por diferentes instituciones, y otor-ga un puntaje a cada país. Para ello utiliza encuestas a expertos (analistas y empresarios) residentes y no residentes en cada país, de manera que se pueda obtener un puntaje promedio; para obtener éste último se estandarizan los datos mediante la técnica de pareamiento por percentiles y, luego, los valo-res obtenidos se someten a una transformación beta. El promedio entre los tres años es el reportado en el Indice de Percepción de Corrupción. Una ex-plicación detallada 
de la construcción del indice, así como datos sobre la confiabilidad de esta medida, se encuentra disponible en http://www.transparency.org.

Como indicador el régimen político se utilizó el puntaje sobre derechos políticos y libertades civiles calculado por Freedom House International y presentado en su informe de 2005 (20), siguiendo la propuesta de Elrich \& Lui (21). Esta institución desde 1970 ha usado encuestas en diferentes países para indagar sobre varios elementos clave para la libertad; el puntaje aquí utilizado tiene un subíndice de derechos políticos y otro de libertades civiles, cada uno con puntajes entre uno y siete en los que los menores deben inter-pretarse como existe mayor libertad. Una descripción más detallada de los métodos de construcción de los índices puede encontrarse en otra publica-ción (20).

La diversidad cultural fue medida con la medida de fraccionalización étnica propuesta por Alesina y colaboradores (22). Esta se fundamenta e interpreta de manera análoga a las medidas tradicionales de fraccionalización; es decir, que es un número entre cero y uno que se puede interpretar como la probabilidad de que dos individuos seleccionados aleatoriamente de una población sean de diferentes grupos étnicos (23). De esta manera los valores que tienden a cero indican una alta homogeneidad y los que tienden a uno a alta heterogeneidad. La selección de esta medida se fundamentó en que in-cluye un mayor número de países que otras similares, permite tener un crite-rio consistente para comparaciones entre países y la alta correlación con otras medidas conocidas de fraccionalización etnolingüística y religiosa. La descripción detallada de la forma en que se construyen estas medidas, así como su validez y posibles usos se encuentran descritos en otro documento (22).

\section{Métodos estadísticos}

En un primer momento se describieron las diferencias en la EVN y los de-más macro-determinantes entre los dos regímenes políticos mediante la prueba $\mathrm{U}$ de Mann-Whitney. Luego se calcularon las correlaciones entre los macrodeterminantes con pruebas de Spearman. Finalmente se realizaron re-gresiones lineales robustas entre el régimen político y al EVN, ajustando por los demás macro-determinantes. Estas regresiones permiten obtener estima-ciones precisas sin cumplir los supuestos de la regresión lineal convencional. Esta técnica inicia estimando una regresión lineal y calcula las distancias de Cook para cada observación; luego elimina del análisis las observaciones más extremas con una distancia de Cook mayor de uno, y finaliza realizando una 
nueva estimación mediante iteraciones de Huber y biponderadas (24), de acuerdo a las distancias de Cook. Todos estos análisis fueron realizados con el programa estadístico Stata 9 (Stata Corporation, College Station, Texas).

\section{RESULTADOS}

En la figura 1 se puede observar que hay diferencia en la EVN cuando se comparan los países presidenciales y los parlamentarios ( $p=0,02)$ que favorece a los últimos; entre los países con régimen presidencial la mediana de la EVN fue de 70 años (rango intercuartil: 8 años), mientras que entre los paí-ses con parlamento fue de 75 años (rango intercuartil: 9 años).

Figura 1. Distribución de la esperanza de vida al nacer en los países con regímenes presidencial y parlamentario $(n=63)$

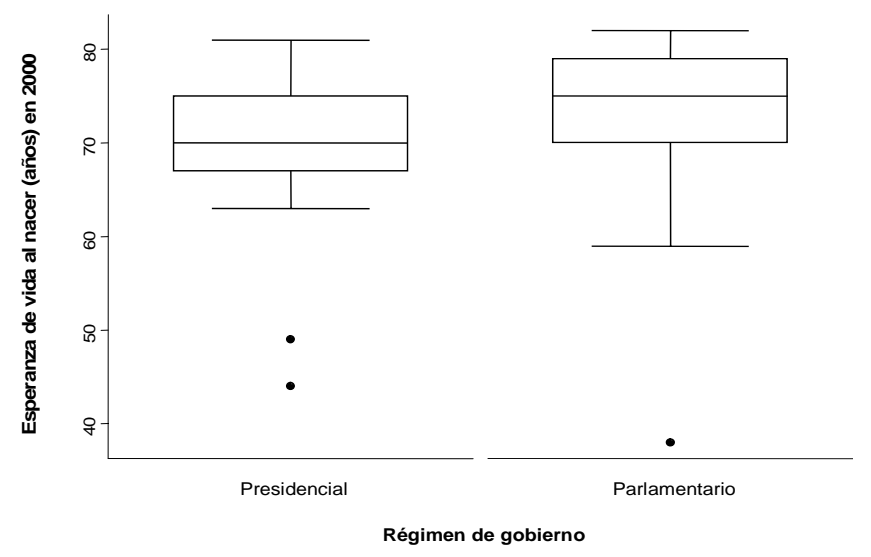

Al comparar los dos regímenes políticos se observaron diferencias importantes en relación a todos los otros macro-determinantes. La mediana del PIB/ ppc fue mayor entre los países con régimen presidencial que entre los de régimen parlamentario $(\mathrm{p}=0,055)$, similar a lo que se observa con el coefi-ciente de Gini $(p<0,0001)$, la percepción de corrupción $(p=0,002)$ y la di-versidad cultural $(p=0,021)$ que también fueron mayores entre los países presidencialistas. De manera contraria, la confianza generalizada fue mayor entre los países parlamentarios ( $<<0,0001)$. En la tabla 1 se encuentran las de Spearman entre los macro-determinantes medidos en escala continua. Nó-tese que todas las variables tienen correlaciones estadísticamente significati-vas ( $p<0,05)$, excepto el PIB/ppc con el coeficiente de Gini y el PIB/ppc y el índice de derechos políticos y libertades civiles. Esto es evidencia de que estos determinantes se encuentran relacionados entre sí o que existe un de-terminante 
común de todos estos. En la tabla 2 se observan los coeficientes de las regresiones robustas donde se ajustó la relación entre la EVN y el ré-gimen político por los otros macro-determinantes. Lo que resulta más evi-dente es que el tener un régimen parlamentario se asocia con mayores EVN sólo cuando se ajusta exclusivamente con el PIB/ppc (modelo 2) o éste, el coeficiente de Gini y el índice de derechos políticos y libertades civiles con-juntamente (modelo 4).

Tabla 1. Correlaciones de Spearman entre algunos de los macro-determinantes estudiados

\begin{tabular}{|c|c|c|c|c|c|c|}
\hline & $n$ & $\begin{array}{l}\text { Coeficiente } \\
\text { de Gini }\end{array}$ & IDPLC & $\begin{array}{c}\text { Indice de Percepción } \\
\text { de Corrupción }\end{array}$ & $\begin{array}{c}\text { Confianza } \\
\text { generalizada }\end{array}$ & $\begin{array}{c}\text { Fraccionalización } \\
\text { étnica }\end{array}$ \\
\hline PIB/ppc & & 0,05 & $-0,20$ & $0,32^{*}$ & $-0,01$ & $-0,26^{*}$ \\
\hline Coeficiente de Gini & & & $0,24^{*}$ & $-0,26^{*}$ & $-0,44^{*}$ & $0,32 *$ \\
\hline IDPLC & & & & $-0,68^{*}$ & $-0,25^{*}$ & $0,37 *$ \\
\hline $\begin{array}{l}\text { Indice de Percepción } \\
\text { de Corrupción }\end{array}$ & & & & & $0,44^{*}$ & $-0,44^{*}$ \\
\hline $\begin{array}{l}\text { Confianza } \\
\text { generalizada }\end{array}$ & & & & & & $-0,27^{*}$ \\
\hline
\end{tabular}

\section{DISCUSION}

El análisis empírico aquí presentado no muestra diferencias importantes entre el régimen presidencialista y el régimen parlamentario en términos del indicador de salud seleccionado. La aparente mayor EVN observada inicialmente parece estar confundida por otros macro-determinantes como los incluidos en el análisis es-tadístico. Esto se hizo evidente al encontrar que los países con presidente tienden a tener un mayor PIB/ppc, una mayor desigualdad en el ingreso, una mayor co-rrupción, una menor confianza generalizada y una mayor diversidad cultural. En términos estadísticos, sólo se observaron efectos benéficos del régimen parla-mentario sobre la EVN en dos de los modelos más simples, y no así en los otros modelos en que se buscó un mayor control. Sin embargo, estos modelos no son directamente comparables entre sí ya que tienen diferentes muestras, debido a la disponibilidad de los datos; se prefirió hacer este tipo de análisis para tratar de mostrar una panorámica lo más completa posible.

El encontrar diferencias importantes entre los dos regímenes en relación a los otros macro-determinantes y correlaciones significativas entre la gran mayoría de éstos últimos sugiere que hay tendencias que caracterizan ambos regímenes, pero en ningún momento indican una relación causal entre éstos; es decir, que no se puede afirmar queal cambiar de un régimen presidencial a uno parlamentario se mejoren los otros macro-determinantes, ni tampoco lo contrario. 


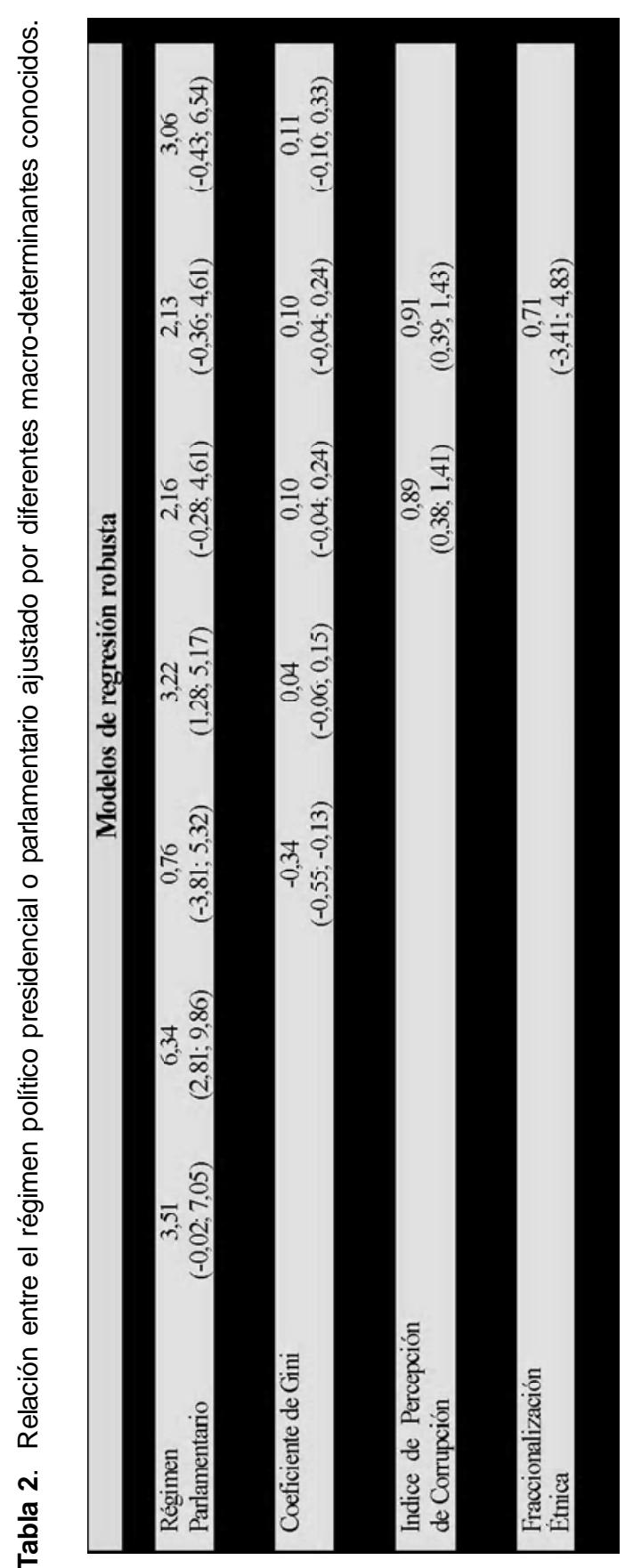


Parece ser más lógico pensar que hay un determinante común del tipo de régimen político y los demás macro-determinantes, no identificado en el presente análisis explora-torio.

En conclusión, la aparente diferencia en la EVN que favorece a los países con regímenes parlamentarios parece estar confundida por otros macrodeterminantes, y que al intentar controlar esta confusión las diferencias con el régimen presidencial se minimizan. Parte de estos hallazgos pueden ser debidos al reducido tamaño de muestra, pero infortunadamente no conocemos otras fuen-tes que permitan incluir un mayor número de países. La evidencia disponible no permite identificar que el cambiar de un régimen presidencial a uno parlamenta-rio resulte en mejorías substanciales en la salud poblacional, por lo que desde el punto de vista de la salud pública puede resultar mucho más efectivo enfocarse a otros macro-determinantes como la desigualdad en el ingreso, que es el que has-ta ahora ha mostrado tener mayores efectos en la salud poblacional*

\section{REFERENCIAS}

1. Linz JJ. The perils of presidentialism. J. Democr. 1990;1:51-69.

2. Linz JJ. The virtues of parliamentarism. J. Democr. 1990;1:84-91.

3. Przeworski A, Alvarez ME, Cheibub JA, Limongi F. Democracy and development: political institutions and well-being in the world, 1950-90. New York: Cambridge Uni-versity Press; 2000.

4. Shugart MS, Carey JM. Presidents and assemblies: Constitutional design and electoral dynamics. New York: Cambridge University Press; 1992.

5. Franco A, Alvarez-Dardet C, Ruiz MT. Effect of democracy on health: ecological study. Br. Med. J. 2004;329:1421-3.

6. Moore S, Teixeira AC, Shiell. The health of nations in a global context: trade, global stratification, and infant mortality rates. Soc. Sci. Med. 2006;63:16578.

7. Shandra JM, Nobles J, London B, Williamson JB. Dependency, democracy, and infant mortality: a quantitative, cross-national analysis of less developed countries. Soc. Sci. Med. 2004;59:321-33.

8. Coburn D. Beyond the income inequality hypothesis: class, neo-liberalism, and health inequalities. Soc. Sci. Med. 2004;58:41-56.

9. Morgenstern H. Ecologic studies in epidemiology: concepts, principles, and methods. Annu. Rev. Public Health 1995;16:61-81.

10. McCann JC. A technique for estimating life expectancy with crude vital rates. Demography 1976;13:259-272. 
11. Almeida-Filho N. O conceito de saúde: ponto-cego da epidemiologia? Rev. Bras. E-pidemiol. 2000; 3:4-20.

12. World Health Organization. World Health Report 2004: Cultural liberty in today's diverse world. Geneva: WHO; 2004.

13. Lawrence CN. Regime stability and presidential government. The legacy of authoritarian rule, 1951-90. Disponible en: http://www.cnlawrence.com/papers/ stability-review.pdf. Consultado: 8 de Julio de 2006.

14. Lawrence CN, Hayes JR. Regime stability and presidential government: A preliminary analysis. Presented at the 2000 Meeting of the Southern Political Science Associa-tion; 2000.

15. United Nations Development Programme. World Report 2004. Cultural liberty in to-day's diverse world. New York: UNDP; 2004.

16. Milanovic B. A simple way to calculate the Gini coefficient, and some implications. Econ. Lett. 1997;546:45-9.

17. Bjørnskov C. The multiple facets of social capital. Eur. J. Pol. Econ. 2006;22:2240.

18. Idrovo AJ. Desigualdad en el ingreso, corrupción y esperanza de vida al nacer en México. Rev Salud Pública (Bogotá) 2005;7:121-9.

19. Transparency International. Transparency International corruption perceptions index. Berlin: Transparency International Secretariat; 2004.

20. Freedom House. Freedom in the World 2005: civic power and electoral politics. New York: Freedom House; 2005.

21. Elrich I, Lui F. Bureaucratic corruption and endogenous economic growth. J. Pol. Econ. 1999;107:s270-s93.

22. Alesina A, Easterly W, Kurlat S, Wacziarg R. Fractionalization. J. Econ. Growth 2003;8:155-94.

23. Easterly W, Levine R. Africa's growth tragedy policies and ethnic divisions. Quart. J. Econ. 1997;112:1203-50.

24. Huber PJ. Regression. In: Robust statistics. New York: John Wiley \& Sons; 1981: 153-198. 